\title{
Christian Schmidt, La Théorie des jeux. Essai
} d'interprétation

PUF, 2001

Sébastien Cochinard

\section{(2) OpenEdition}

\section{Journals}

Édition électronique

URL : http://journals.openedition.org/ei/728

DOI : $10.4000 /$ ei. 728

ISSN : 2553-1891

\section{Éditeur}

Association Économie et Institutions

Édition imprimée

Date de publication : 1 juin 2003

Pagination : 167-169

ISSN : 1775-2329

\section{Référence électronique}

Sébastien Cochinard, "Christian Schmidt, La Théorie des jeux. Essai d'interprétation », Économie et institutions [En ligne], 2 | 2003, mis en ligne le 31 janvier 2013, consulté le 23 septembre 2020. URL http://journals.openedition.org/ei/728 ; DOI : https://doi.org/10.4000/ei.728 


\title{
Commentaire de l'ouvrage de Christian Schmidt : La Théorie des jeux. Essai d'interprétation, PUF, 2001.
}

\author{
Sébastien Cochinard, Université de Picardie
}

Christian Schmidt nous invite à parcourir plus d'un demisiècle de recherches en théorie des jeux, avec le regard critique de l'économiste, de l'historien et du philosophe des sciences. Tenant à la fois de la considération analytique et de l'essai interprétatif, l'ouvrage interroge le passé pour mieux mettre en questions le présent et sonder le futur de cette discipline aux multiples facettes (la justesse même des termes "théorie des jeux" y est commentée dans l'Introduction générale).

Une première partie étudie l'unité de la théorie des jeux. Multiplicité, caractère contre-intuitif voire absence des équilibres sont abordés par l'intermédiaire d'exemples célèbres tels que celui du dilemme du prisonnier. A travers celui du jeu de la "poule mouillée ", l'auteur évoque (p.45) les thèmes de l'équilibre corrélé, de l'équilibre bayésien ou encore de la purification d'équilibre en stratégies mixtes (par la référence à Harsanyi, 1973). L'exemple se serait prêté à une comparaison détaillée de ces approches complémentaires proposées par la théorie des jeux. L'exposé des différentes versions du jeu de la chasse aux cerfs, dont Christian Schmidt met en cause la fidélité à l'œuvre de Rousseau, permet le réexamen du thème de la coordination des joueurs dans des situations d'équilibres multiples.

Le choix du concept d'équilibre forme le thème de la deuxième partie du livre ("Questionnement Un"). L'auteur distingue les différents niveaux d'intervention de la théorie des probabilités en jeux, qu'elles soient objectives ou subjectives, qu'elles représentent la manifestation d'un aléa externe, l'effet d'une randomisation volontaire de la part des joueurs ou l'expression de croyances réciproques entre adversaires. A travers le jeu "ciseaux, pierre, papier " (p.84 et suivantes), présenté comme le jeu des navires sous forme de jeu à somme constante (et non nulle, p.106), Christian Schmidt souligne le saut cognitif (p. 125) qui autorise les théoriciens des jeux à utiliser l'extension mixte d'un jeu. Reconnaissons cependant qu'en l'absence du concept d'équilibre de Nash en stratégies mixtes qui lui est associé, la théorie des jeux se trouverait fort démunie. Si l'approche par la purification permet de contourner élégamment l'utilisation des stratégies mixtes, son utilisation pratique (si l'on ose dire) en théorie des jeux est restée limitée.

Reprenant les liens unissant théorie de la décision et théorie des jeux, Christian Schmidt développe la problématique de la 
dissuasion et de l'incitation à travers la distinction entre stratégies directes et indirectes. Ces dernières amènent en fait à l'adjonction au jeu initial de jeux annexes (solution maintes fois utilisée par la théorie des jeux), dont on pourra objecter le caractère ad hoc. Les stratégies indirectes introduisent l'usage des jeux sous forme extensive et la discussion détaillée du rôle particulier joué par la "backward induction" dans cette forme de jeux. Les stratégies de comportement de Kuhn, si elles sont clairement évoquées en tant que concept historique et naturel dans cette forme de jeu, auraient pu être reliées explicitement aux problématiques de l'équilibre de Nash mixte en jeux sous forme stratégique ou de la révision dynamique des croyances au cours du déroulement du jeu. L'auteur discute enfin les fondements épistémiques de l'équilibre de Nash, eu égard notamment à la nature de la rationalité présupposée des joueurs.

La troisième partie du livre ("Questionnement Deux") est dédiée à l'analyse de ce qui est souvent présenté ailleurs comme les deux versants de la théorie - jeux coopératifs et jeux non-coopératifs - mais dont Christian Schmidt, sous le nom de "co-opération", relève le caractère commun touchant à la coordination des actions des joueurs. Un bref détour par la théorie des jeux évolutionnaires permet à l'auteur de situer le " programme de Nash ", qui subsume la coopération à la non-coopération, et d'en cerner les caractéristiques distinctives face au projet originel de von Neumann et Morgenstern. Les jeux sous forme coalitionnelle (c'est-à-dire coopératifs) sont ensuite présentés avec leurs concepts de solution centraux de cœur et de valeur de Shapley. Christian Schmidt analyse enfin les rapports qu'entretiennent les différents modèles (stratégique, coalitionnel et extensif) et questionne leurs présupposés en termes d'information et de connaissance des joueurs. La théorie des jeux évolutionnaires (évoquée p.217 et 371) aurait sans doute mérité de figurer en tant que quatrième "forme " de jeu (p. 329 et 354) à part entière. Les problématiques de cette discipline auraient alors été développées pour elles-mêmes : la construction de concepts d'équilibres allant au-delà de l'ESS, ses applications économiques (par exemple, en théorie de la fiscalité publique dans les travaux de Weibull) ou encore les liens qu'entretient cette théorie et plus généralement l'évolutionnisme en science économique.

Dans une quatrième partie à la tonalité plus historique, l'auteur situe les rôles respectifs de Borel, Zermelo puis de Cournot et Edgeworth, dans une vision qui tranche avec la présentation d'une histoire orthodoxe et linéaire de la théorie des jeux. Le statut spécifique de l'expérimentation en théorie des jeux y est aussi cerné dans sa complexité. L'auteur termine cette partie par l'exposé de la 
controverse entre Aumann et Binmore sur les liens unissant "backward induction " et connaissance commune de la rationalité, ce qui permet de donner un exemple des enjeux scientifiques propres à la théorie.

Christian Schmidt clôt son ouvrage par une référence leibnizienne au rôle spécifique du jeu dans une théorie de la connaissance. Il nous invite à poursuivre la réflexion dans la direction d'une meilleure description et d'une compréhension plus complète des fondements épistémologiques des interactions multiples à l'œuvre dans les jeux.

Fourmillant d'exemples analysés en détail, à mi-chemin entre l'introduction et l'essai, l'ouvrage de Christian Schmidt ne laissera indifférent ni le néophyte, ni l'auteur éclairé ou encore le spécialiste des questions ludiques. Au premier, il donnera l'occasion de découvrir, par une présentation pédagogique, un domaine de recherches étonnant par l'objet visé : l'interaction. Au second, il permettra de resituer ses connaissances dans l'étendue du champ couvert par la théorie des jeux : économie, gestion, mathématiques, sciences politiques, biologie, philosophie, sociologie, psychologie, informatique théorique, sciences cognitives, etc. Au dernier, il permettra la confrontation avec une pensée hétérodoxe par les bases qu'elle remet en cause et par sa manière de présenter les idées. 